\title{
Coplanar-Waveguide-Fed Microstrip Bandpass Filters With Capacitively Broadside-Coupled Structures for Multiple Spurious Suppression
}

\author{
Chi-Hsueh Wang, Member, IEEE, Pu-Hua Deng, and Chun Hsiung Chen, Fellow, IEEE
}

\begin{abstract}
Coplanar-waveguide (CPW)-fed microstrip bandpass filters are proposed with capacitive couplings suitably introduced at the input/output (I/O) ports, as well as between the resonators for spurious suppression. By adopting these capacitive couplings, several open stubs are established so that adjustable multiple transmission zeros may independently be created to suppress several unwanted spurious passbands, thereby extending the stopband and improving the rejection level. In this study, the capacitive couplings required at the $I / O$ ports, as well as across the resonators, are realized by the broadside-coupled transition structures between the top microstrip layer and the bottom CPW layer so that the I/O ports may properly be matched and the spurious responses may effectively be suppressed. Specifically, a fifth-order bandpass filter, centered at $f_{0}=1.33 \mathrm{GHz}$ with a stopband extended up to $8.67 \mathrm{GHz}\left(6.52 f_{0}\right)$ and a rejection level better than $30 \mathrm{~dB}$, is implemented and carefully examined.
\end{abstract}

Index Terms-Bandpass filter, coplanar waveguide (CPW), dual metal-layer structure, microstrip, spurious suppression.

\section{INTRODUCTION}

$\mathbf{I}$ $\mathrm{N}$ MICROWAVE communication systems, the filters with deep stopband and good selectivity are required to enhance the system performance. In order to reduce the interference by keeping the out-band signals from reaching a sensitive receiver, a high-performance filter with wider upper stopband is also required. However, the planar bandpass filters made of half-wavelength $(\lambda / 2)$ uniform-impedance resonators have unwanted spurious passbands at the multiples of center frequency $n f_{o}(n=2,3, \ldots)$, where $f_{o}$ is the passband center frequency. Several filters using the stepped-impedance resonators were proposed to shift the spurious passbands to the higher frequencies so as to extend the upper rejection band [1]-[3]. However, the stepped-impedance method can only push the spurious passbands to the higher frequencies instead of suppressing them. If one would like to move the first spurious passband to $5 f_{0}$, e.g., by using the $\lambda / 2$ stepped-impedance resonators, a large impedance ratio for the resonators is required such that the layout of the filter becomes difficult due to the fabrication limit.

Manuscript received August 18, 2006; revised October 26, 2006. This work was supported by the National Science Council of Taiwan under Grant NSC 94-2752-E-002-001-PAE, Grant NSC 94-2219-E-002-008, and Grant NSC 94-2213-E-002-055.

The authors are with the Department of Electrical Engineering and Graduate Institute of Communication Engineering, National Taiwan University, Taipei 106, Taiwan, R.O.C. (e-mail: chchen@ew.ee.ntu.edu.tw).

Digital Object Identifier 10.1109/TMTT.2007.892816
To solve these drawbacks, several methods to suppress spurious passbands have been reported [4]-[12]. In [4], the "wiggly-line" filters using a sinusoidally varying linewidth were proposed to give an effective suppression on the first spurious response around $2 f_{0}$. Corrugated coupled microstrip lines [5]-[7] that are designed to equalize the phase velocities of the two eigenmodes were adopted to design the planar filters with suppression of the first spurious passband. In [8], an over-coupled resonator was employed to increase the electrical length of the odd mode so as to compensate for the difference in the phase velocities. Alternatively, the ideas of proposing substrate suspension [9] and ground-plane aperture [10] were reported to equalize the even- and odd-mode phase velocities so that the first spurious response can be suppressed. By etching split-ring resonators [11] to achieve strong magnetic coupling, the first spurious passband may also be suppressed. In [12], a meandered parallel coupled-line structure was utilized to suppress the first spurious passband of a microstrip bandpass filter. However, most of these filter configurations still suffer from the higher order spurious responses around $n f_{0}(n=3,4, \ldots)$ [4]-[12].

The concept of using dissimilar resonators to produce different harmonic frequencies for each resonator was proposed [13]-[15]. Based on this concept, one may implement a widestopband microstrip bandpass filter for which the spurious responses may be suppressed due to the mutual cancellation effect. However, the technique of adopting dissimilar resonators is not feasible in controlling the bandwidth of stopband and it would also suffer from the difficulty of choosing the proper dissimilar resonators to avoid the mutual coupling effects in the higher order filter design.

Recently, a coplanar waveguide (CPW) bandpass filter with rejection band extended up to $19 f_{o}$ was reported [16]. This filter is mainly composed of two parts, i.e., the loaded air-bridge enhanced capacitors to suppress the lower order spurious passbands and the broadside-coupled microstrip-to-CPW-fed structures to attenuate the high-order spurious responses, therefore, its stopband may be extended even up to $19 f_{o}$. Although the method adopted by [16] is useful in realizing a very-wide stopband CPW filter, it is difficult to extend to the other structures using different configurations.

In [17] and [18], capacitive-tapped couplings between resonators and input/output (I/O) were established so that extra transmission zeros in the stopband can easily be created without requiring complex couplings between resonators. Here, tapped chip capacitors were inserted between the resonators, thus the 
implementation process becomes complicated and the discontinuity effect may be produced. In [19], by controlling the locations of I/O tapped points of the parallel-coupled filter, the spurious passband was cancelled by inserting a notch at the spurious frequency. However, two additional quarter-wavelength $(\lambda / 4)$ transformers are required at the I/O ports of the filter in [19], thereby increasing the circuit area.

In this study, the microstrip bandpass filters composed of $\lambda / 2$ resonators (such as the one shown in Fig. 1) with capacitive couplings for multiple spurious suppression are proposed and carefully examined. First, the I/O capacitive couplings are achieved by using the broadside-coupled CPW-to-microstrip transition structures [20] to replace the two I/O $\lambda / 4$ matched transformers adopted in [19] so that the I/O ports may properly be matched and the filter size may be reduced. These two I/O capacitive-coupled structures may also provide two open stubs to create two transmission zeros for spurious suppression. Second, the capacitive couplings between the adjacent resonators are realized by the broadside-coupled microstrip-to-CPW-to-microstrip transition structures instead of the parallel-coupled-line structures used in [19] so that extra open stubs may be created to produce additional transmission zeros for spurious suppression.

At first glance, the proposed filter (Fig. 1) might look similar to the dual-behavior resonator (DBR) filter discussed in [21]; however, they are quite different in topology and function. Briefly, the DBR filters are based on the parallel association of two stopband structures which are carefully designed to create two transmission zeros on either side of the passband. By using the stepped-impedance and different-length stubs, the lengths of associated open or shorted stubs may be adjusted independently, thus the created transmission zeros may be controlled freely. The main drawback of DBR filters concerns the attenuated band which is difficult to control over a wide range of frequencies, and this drawback may be solved by some special techniques [21]. The proposed filter topology is originated from the hairpin coupled-resonator filter. By using the capacitively broadside-coupled structures for feeding and coupling, several open stubs can freely be established so as to create adjustable multiple transmission zeros for suppressing several unwanted spurious responses, therefore, the rejection level can be improved and the stopband can be extended even higher than $6 f_{0}$, as will be demonstrated later. Moreover, the lengths and other parameters of open stubs may be adjusted, thus one may easily control the locations and widths of the attenuation dips in the filter response.

In comparison with the techniques used in [13]-[15] and [16], the method proposed in this study is easy in extension and simple in controlling the bandwidth of stopband. The proposed method would have a limitation in implementing a wide stopband filter, as did the one in [16], due to the difficulty in realizing the open stubs of very short lengths.

In this study, the basic principle of spurious suppression is demonstrated by implementing a second-order microstrip bandpass filter, which has four open stubs to create four transmission zeros for suppression of spurious passbands. Specifically, a fifth-order microstrip bandpass filter is implemented, having ten open stubs to create five pairs of transmission zeros. Each

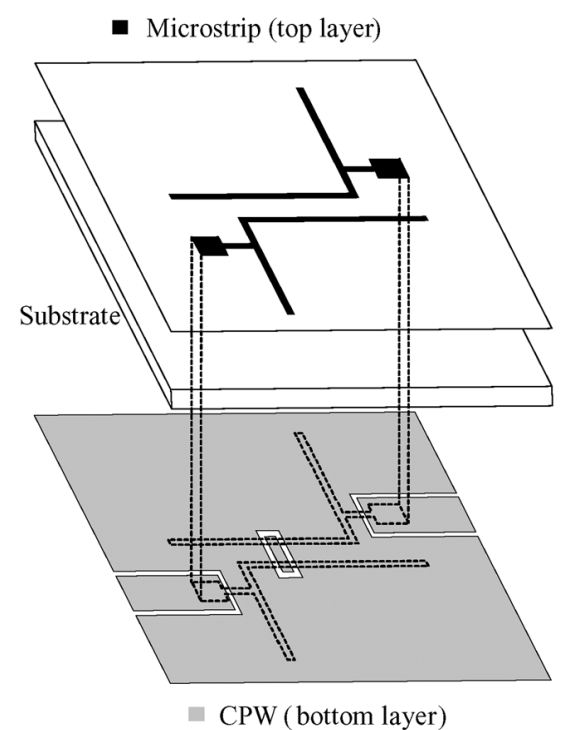

(a)

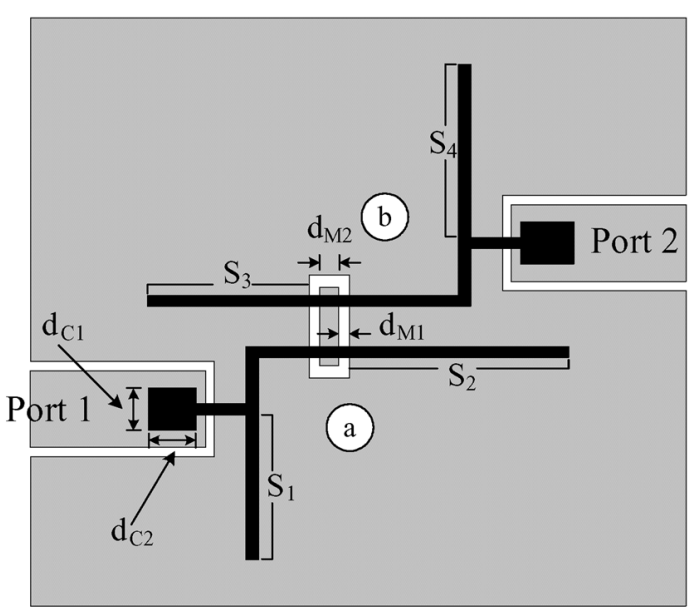

(b)

Fig. 1. Proposed second-order bandpass filter with capacitively broadside-coupled structures for feeding and coupling. (a) 3-D circuit layout. (b) Top-/bottomlayer circuit layouts to show the relative location between top microstrip layer and bottom CPW layer.

pair of transmission zeros is designed to suppress one spurious passband; thus, the implemented fifth-order filter may suppress five spurious passbands.

\section{SECOND-ORDER FILTERS}

\section{A. Filter Structure and Design}

The proposed second-order coupled-resonator microstrip bandpass filter structure composed of $\lambda / 2$ resonators is shown in Fig. 1 in which capacitively broadside-coupled structures are utilized to establish the proper feed circuits for I/O ports and the required couplings between the adjacent resonators. The relative location between the top microstrip layer and bottom CPW layer is shown in Fig. 1(b). Here, the broadside-coupled CPW-to-microstrip transition structures are employed to realize the I/O capacitive couplings instead of using two $\lambda / 4$ transformers in [19] so that the I/O ports may properly be matched and the filter size may be reduced. The I/O coupled structures 


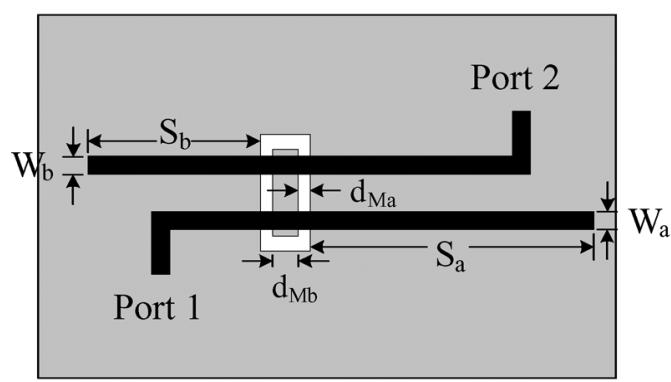

Fig. 2. Capacitively broadside-coupled microstrip-to-CPW-to-microstrip transition structure and the associated open stubs of lengths $S_{a}$ and $S_{b}$. ( $W_{a}=$ $W_{b}=1.2 \mathrm{~mm}, d_{M a}=0.4 \mathrm{~mm}$, and $d_{M b}=0.9 \mathrm{~mm}$ ).

provide two open stubs $\left(S_{1}, S_{4}\right)$ for producing two transmission zeros. The coupling between resonators $a$ and $b$ is realized by the capacitively broadside-coupled microstrip-to-CPW-to-microstrip transition structure, which also supplies two open stubs $\left(S_{2}, S_{3}\right)$ for spurious suppression. Being composed of $\lambda / 2$ resonators, the proposed filter in Fig. 1 would exhibit the spurious responses around $n f_{0}(n=2,3, \ldots)$.

The open stubs, such as $S_{1}, S_{2}, S_{3}$, and $S_{4}$ in Fig. 1, are mainly introduced for spurious suppression. To this end, each open stub should properly be designed such that its length is equal to $\lambda / 4$ at the spurious frequency $f_{s}\left(=n f_{0}\right)$; therefore, it would create a transmission zero designed to suppress the spurious passband around $f_{s}$. The width of the open stub, which is related to the impedance of the transmission line in the stub, can determine the width and sharpness of the attenuation dip associated with the filter response in the stopband. For design purposes, the response to the broadside-coupled microstrip-toCPW-to-microstrip transition structure shown in Fig. 2 needs to be investigated. Typical design curves presenting the relations between the transmission-zero frequencies and the lengths $S_{a}$ and $S_{b}$ of open stubs are shown in Fig. 3(a) and (b). Based on these design curves, the proper stub lengths $S_{a}$ and $S_{b}$ to locate the transmission zeros around $n f_{0}$ may then be determined.

The capacitive coupling between adjacent resonators, such as resonators $a$ and $b$, is established by the broadside-coupled microstrip-to-CPW-to-microstrip transition structure, as depicted in Fig. 1. The corresponding coupling coefficient would specify the required coupling capacitance between the two adjacent resonators, thereby determining the associated broadside-coupled structure between resonators. For example, the coupling coefficient $M_{a b}$ of the filter in Fig. 1 may be adjusted by varying the coupling capacitor between resonators $a$ and $b$ with its capacitance value mainly decided by the width $d_{M 2}$ in Fig. 1(b). The design curve for $M_{a b}$ versus $d_{M 2}$ is shown in Fig. 4 for further reference.

To design the proposed bandpass filter (Fig. 1) with its passband center frequency at $f_{0}$, each $\lambda / 2$ resonator is selected so that its resonant frequency is equal to the specified center frequency $f_{0}$. Note that the lengths of open stubs $S_{1}$ and $S_{2}$ have already been decided by the spurious frequencies $f_{s}\left(=n f_{0}\right)$ under rejection. To make sure that resonator $a$ in Fig. 1 is resonated at the specified center frequency $f_{0}$, the remaining length extracting $S_{1}$ and $S_{2}$ should be chosen such that the total length of resonator $a$ is equal to $\lambda / 2$ at $f_{0}$. Obviously, through using

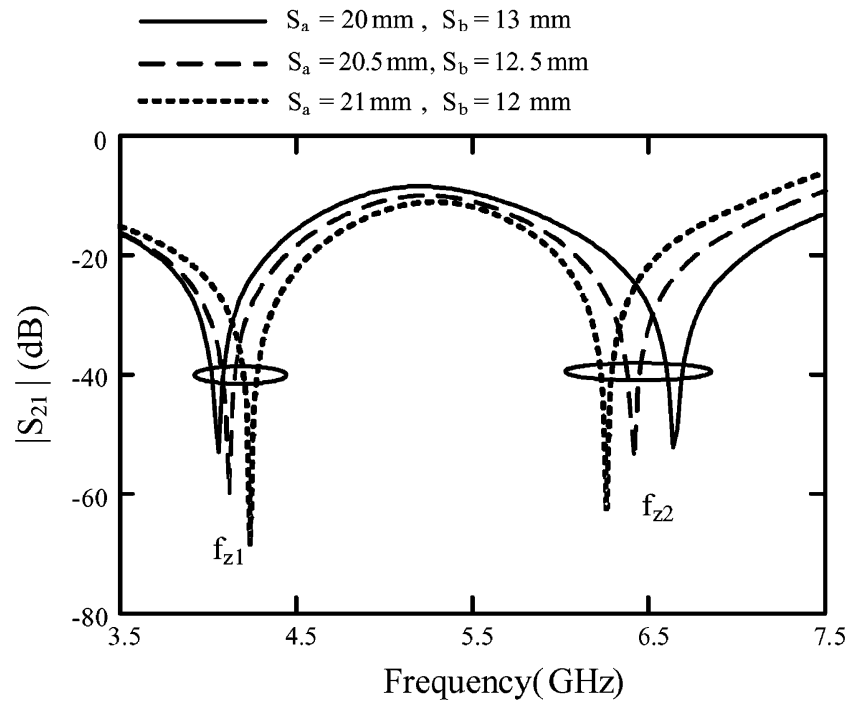

(a)

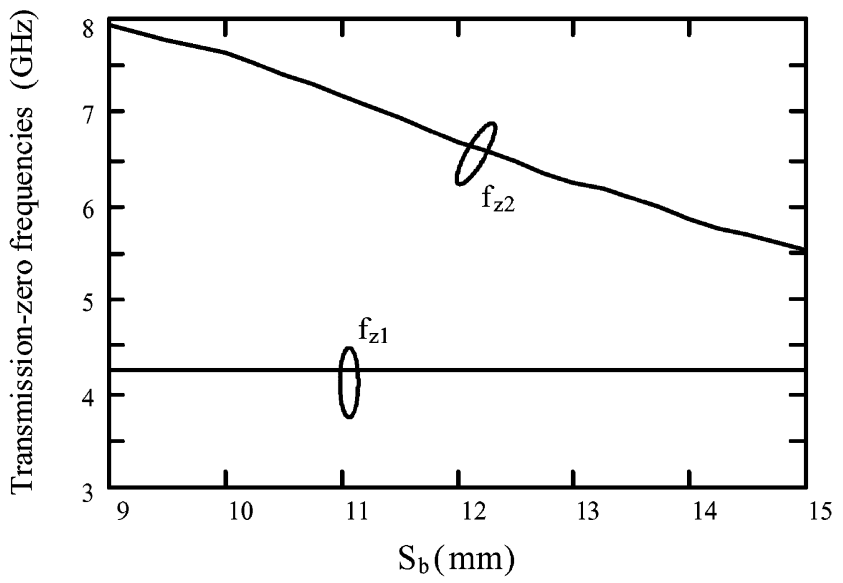

(b)

Fig. 3. (a) Full-wave simulated responses of the capacitively broadside-coupled transition structure in Fig. $2\left(W_{a}=W_{b}=1.2 \mathrm{~mm}\right.$ ). (b) Design curves to relate the transmission-zero frequencies $f z_{1}, f z_{2}$ to the length $S_{b}$ of open stub in Fig. 2. $\left(S_{a}=20 \mathrm{~mm}\right.$.)

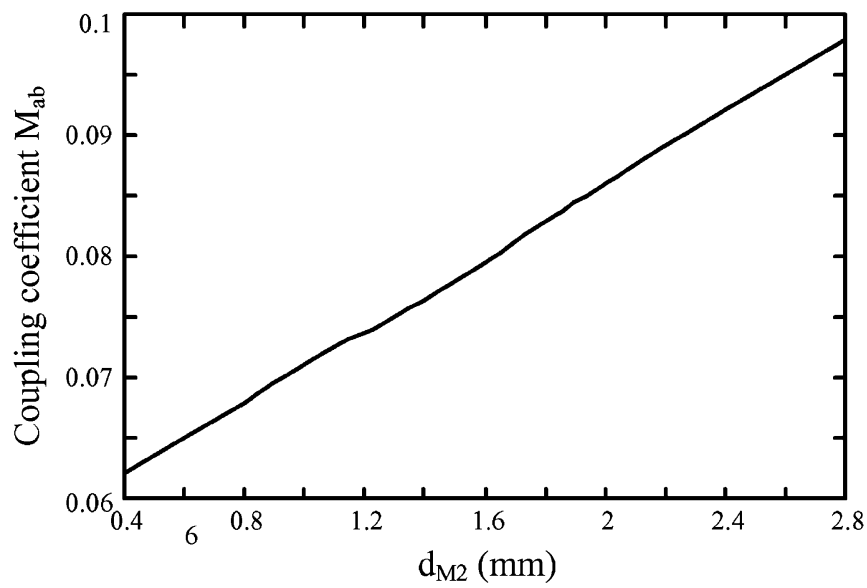

Fig. 4. Coupling coefficient $M_{a b}$ versus the width $d_{M 2}$ [see Fig. 1(b)] of the coupled structure between resonators $a$ and $b .\left(d_{M 1}=0.4 \mathrm{~mm}\right.$.)

the broadside-coupled elements, the choice of the stubs' length is free and not dependent on the design of resonator. Although 


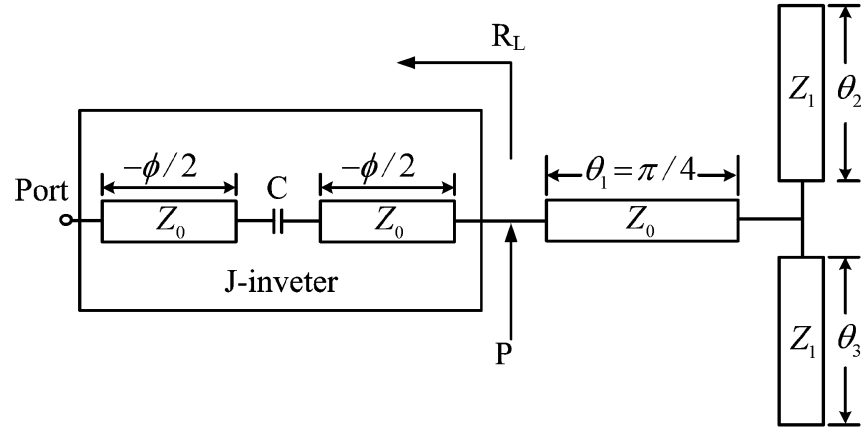

Fig. 5. Feed structure associated with the resonator near I/O port.

the transmission-zero frequencies due to the open stubs are far from the passband, the open stubs still have small influences on the resonance frequency of the resonator. This is why the lengths of open stubs should be decided at the first design step, and, in the meantime, one should make sure that the total length of each resonator is equal to $\lambda / 2$ at $f_{0}$. These small influences will be considered using the transmission line model.

The feed structure associated with the resonator near the I/O port should properly be designed so as to give the specified external quality factor $Q$. The value of the external quality factor would be determined from the filter specification. Fig. 5 shows the proposed feed structure in which a capacitor $C$ and suitable transmission line sections are adopted for I/O coupling and impedance matching. This feed structure is connected to the resonator for which its $Q$ value is given by [19].

The load impedance $R_{L}$, defined at point $P$ of Fig. 5, may be written as

$$
\begin{aligned}
R_{L} & =2 Q\left[\frac{Z_{0} A^{2}}{B(C+D)+A E}\right] \\
A & =Z_{1}-Z_{0} \tan \theta_{1}\left(\tan \theta_{2}+\tan \theta_{3}\right) \\
B & =Z_{1} \tan \theta_{1}+Z_{0}\left(\tan \theta_{2}+\tan \theta_{3}\right) \\
C & =Z_{0}\left(\theta_{2} \sec ^{2} \theta_{2}+\theta_{3} \sec ^{2} \theta_{3}\right) \tan \theta_{1} \\
D & =Z_{0} \theta_{1} \sec ^{2} \theta_{1}\left(\tan \theta_{2}+\tan \theta_{3}\right) \\
E & =Z_{1} \theta_{1} \sec ^{2} \theta_{1}+Z_{0}\left(\theta_{2} \sec ^{2} \theta_{2}+\theta_{3} \sec ^{2} \theta_{3}\right) .
\end{aligned}
$$

If this load impedance $R_{L}$ is not equal to $50 \Omega$, a $\lambda / 4$ transformer or J-inverter should be added for impedance matching [19]. Note that the series coupling capacitor $C$ together with two transmission line sections of characteristic impedance $Z_{0}\left(=1 / Y_{0}\right)$ and length $\phi / 2$ at its two ends may be equivalent to a J-inverter, as shown in Fig. 5, for impedance matching. Consequently, the required I/O coupling (or matching) capacitance $C$ and length $\phi$ may be given by

$$
\begin{aligned}
C & =\frac{Y}{\omega_{0}\left[1-\left(Y / Y_{0}\right)^{2}\right]} \\
Y & =\frac{1}{\sqrt{Z_{0} R_{L}}} \\
\phi & =\tan ^{-1}\left(\frac{2 B_{c}}{Y_{0}}\right)
\end{aligned}
$$

where $B_{C}$ is the susceptance of the capacitance $C$.

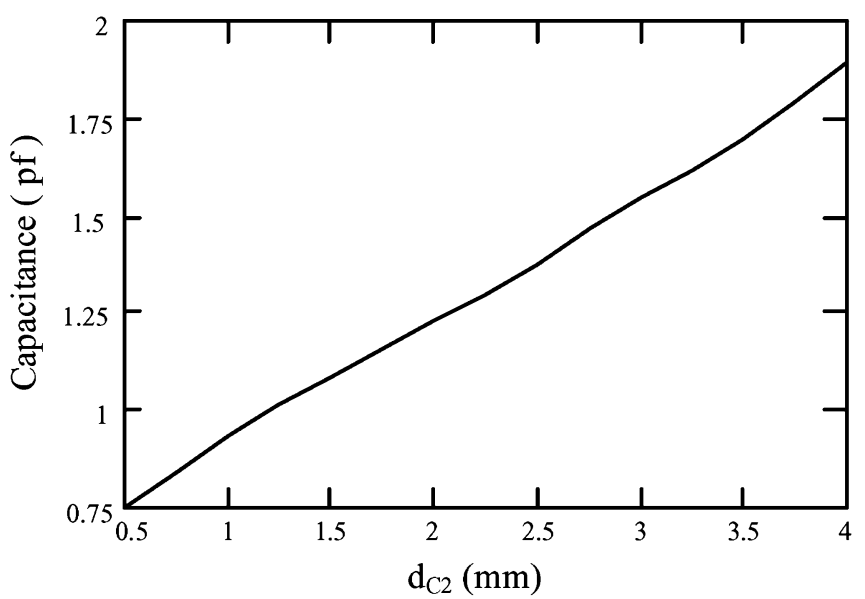

Fig. 6. Coupling capacitance versus the width $d_{C 2}$ [see Fig. 1(b)] of the I/O coupled structure. $\left(d_{c 1}=4.5 \mathrm{~mm}\right.$. $)$

The I/O capacitive coupling is realized by the parallel-plate capacitor structure with a size of $d_{C 1} \times d_{C 2}$, as shown in Fig. 1(b). The coupling capacitance $C$ versus the width $d_{C 2}$ is shown in Fig. 6.

Finally, the broadside-coupled CPW-to-microstrip transition structures (J-inverters) are introduced at the input and output so that the I/O ports may properly be matched.

The design guidelines for the proposed coupled-resonator bandpass filters, such as the one in Fig. 1, are summering in the following.

- The first step is to design each open stub associated with the resonators so that it would create a transmission zero to suppress the spurious passband around $f_{s}\left(=n f_{0}\right)$.

- The second step is to design the filter resonators so as to meet the given specifications.

- The third step is to implement the required coupling coefficients between the adjacent resonators so as to meet the bandwidth specification for the filter.

- The last step is to deal with the feed structure using (1)-(3).

\section{B. Filter Implementation and Results}

The proposed CPW-fed second-order microstrip bandpass filter with capacitively broadside-coupled transition structure between adjacent resonators is fabricated on the Rogers RO4003C substrate $\left(\varepsilon_{r}=3.38, \tan \delta=0.0027\right.$, and thickness $h=0.508 \mathrm{~mm}$ ). Fig. 1 shows the $3-\mathrm{D}$ and top-/bottom-layer layouts of the proposed second-order microstrip bandpass filter. The implemented filter has a dimension of $0.319 \lambda \times 0.42 \lambda$ ( $40.4 \mathrm{~mm} \times 53 \mathrm{~mm}$ ), where $\lambda$ is the guided wavelength of the microstrip structure at the center frequency $f_{0}$. This filter is designed according to the second-order maximally flat response with a center frequency of $1.45 \mathrm{GHz}$ and a 3-dB bandwidth of $12 \%$, for which the coupling coefficient $M_{a b}$ is 0.083 . The specific dimension $d_{M 2}$ [see Fig. 1(b)] to give the required $M_{a b}$ may be determined from Fig. 4.

The external quality factors $Q$ at input and output are 12.8. The required coupling capacitances at input and output are 1.34 and $1.49 \mathrm{pF}$, respectively, and their values may be calculated by (2). The required dimension $d_{c 2}$ [see Fig. 1(b)], which establishes the desired $C$ value is given in Fig. 6. The corresponding 


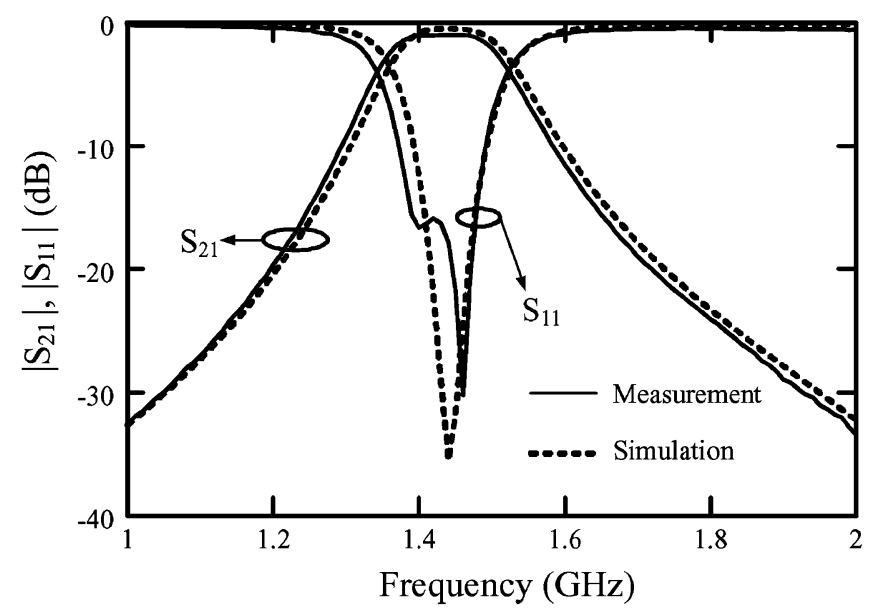

(a)

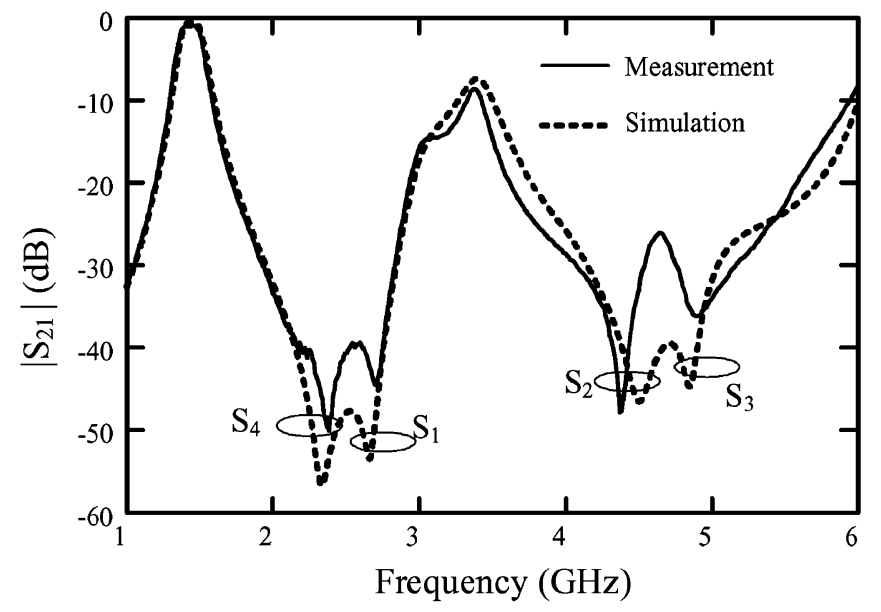

(b)

Fig. 7. Measured and simulated results, insertion loss $\left(\left|S_{21}\right|\right)$, and return loss $\left(\left|S_{11}\right|\right)$ of the proposed second-order filter in Fig. 1. (a) Narrowband and (b) wideband frequency responses.

geometrical parameters are then fine tuned in the full-wave simulator Ansoft Ensemble 8.0.

The measured and simulated results of the implemented filter (Fig. 1) are shown in Fig. 7. The measured center frequency is at $1.43 \mathrm{GHz}$ and the minimum insertion loss is $1 \mathrm{~dB}$. Four transmission zeros are produced by the four open stubs $S_{4}, S_{1}, S_{2}$, and $S_{3}$ (Fig. 1) and are found at 2.33, 2.66, 4.51, and $4.85 \mathrm{GHz}$, respectively. The shift in these transmission-zeros frequencies is less than $5 \%$.

The sensitivity of this proposed filter is mainly dependent on the fabrication process. Normally the fabrication error associated with the size is approximately $\pm 0.0254 \mathrm{~mm}$ and the corresponding electrical length error is $\pm 1^{\circ}$ at $20 \mathrm{GHz}$. Thus, the proposed filter would have a limitation in extending the bandwidth of stopband due to the difficulty in realizing the open stubs of very short lengths.

The second filter structure, shown in Fig. 8, has four transmission zeros designed to suppress the first spurious passband. This implemented filter has a dimension of $0.225 \lambda \times 0.336 \lambda$ $(28.1 \mathrm{~mm} \times 41.9 \mathrm{~mm})$. Specifically, the two transmission zeros associated with the two open stubs $S_{1}$ and $S_{4}$ are distributed around $3.4 \mathrm{GHz}$ and the other two zeros produced by the two

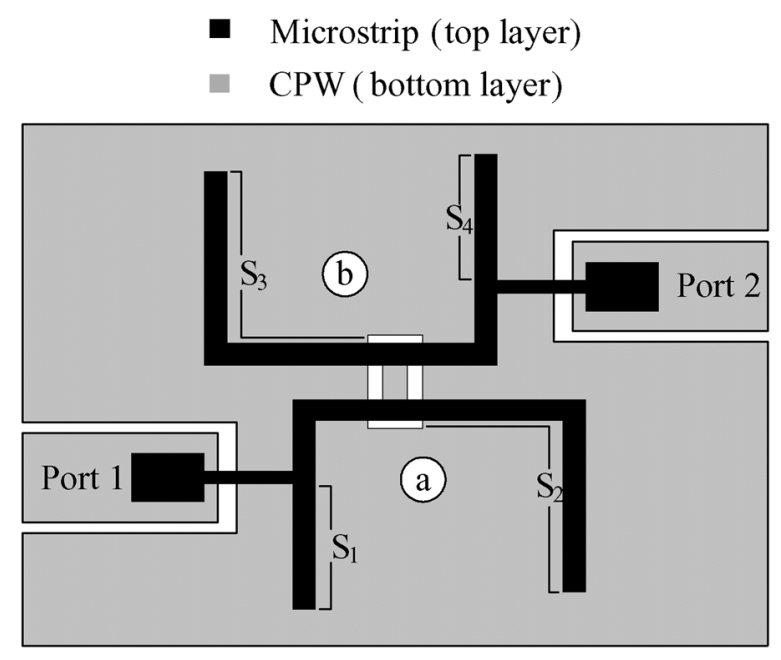

Fig. 8. Top-/bottom-layer layouts of the second-order filter with four transmission zeros to distribute around the first spurious passband.

stubs $S_{2}$ and $S_{3}$ are distributed around $3.6 \mathrm{GHz}$. This filter is also designed according to the second-order maximally flat response with a center frequency of $1.44 \mathrm{GHz}$, a 3-dB bandwidth of $13.5 \%$, and the required coupling coefficient $M_{a b}$ is 0.0954 . The external quality factors $Q$ at input and output are 10.47. The coupling capacitances at input and output are $1.44 \mathrm{pF}$. The measured and simulated results of the second filter (Fig. 8) are shown in Fig. 9. The measured center frequency is at $1.43 \mathrm{GHz}$, the minimum insertion loss is $1.05 \mathrm{~dB}$. The frequency response for the filter in Fig. 1 is also compared with that for Fig. 8. With four transmission zeros designed for the first spurious harmonic, the filter in Fig. 8 has much better rejection around this harmonic, as shown in Fig. 10.

\section{FIFTH-ORDER FILTER}

Fig. 11 shows the top-/bottom-layer layouts of the proposed fifth-order filter. Here, the capacitive couplings at I/O ports are again implemented by the broadside-coupled CPW-to-microstrip transition structures and those between adjacent resonators are by the broadside-coupled-microstripto-CPW-to-microstrip transition structures. This filter has ten open stubs to create ten transmission zeros, which are assigned to some specified frequencies. Specifically, the transmission zeros due to the stubs $\left(S_{1}, S_{9}\right),\left(S_{2}, S_{10}\right),\left(S_{4}, S_{7}\right),\left(S_{3}, S_{8}\right)$, and $\left(S_{5}, S_{6}\right)$ are designed to distribute around the first, second, third, fourth, and fifth spurious harmonics, respectively, so that five spurious passbands may effectively be suppressed.

The design guidelines for this fifth-order filter are similar to the ones for the second-order filter, as mentioned in Section II. The proposed filter is designed according to the fifth-order Chebyshev filter with a $0.1-\mathrm{dB}$ ripple level, having a center frequency of $1.35 \mathrm{GHz}$ and a $3-\mathrm{dB}$ bandwidth of $10 \%$. The required I/O coupling capacitances and coupling coefficients between adjacent resonators are obtained as in Section II, and the corresponding coupling coefficients $M_{a b}, M_{b c}, M_{c d}$, and $M_{d e}$ are $0.077,0.0583,0.0583$, and 0.077 , respectively. The external quality factors $Q$ at input and output are 11.8. The coupling capacitances at input and output are $1.51 \mathrm{pF}$. 


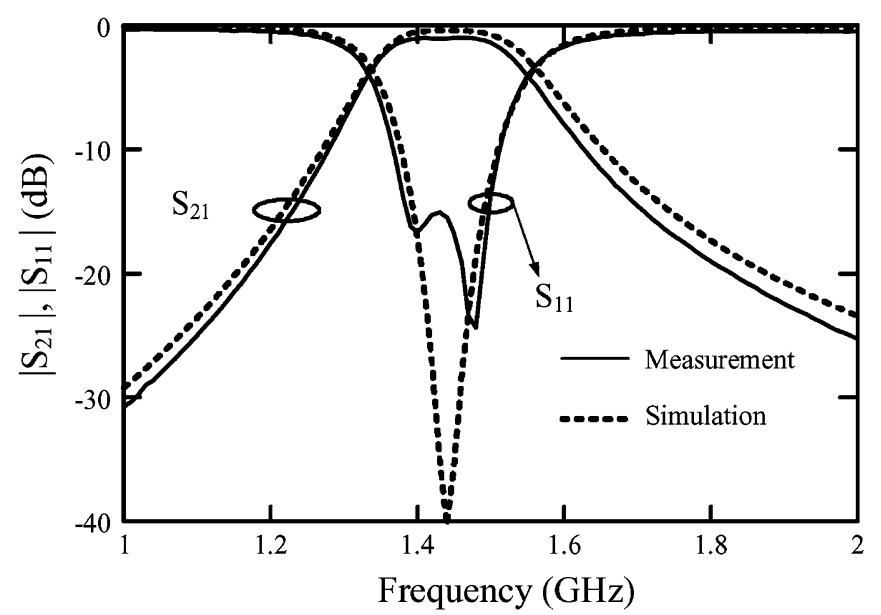

(a)

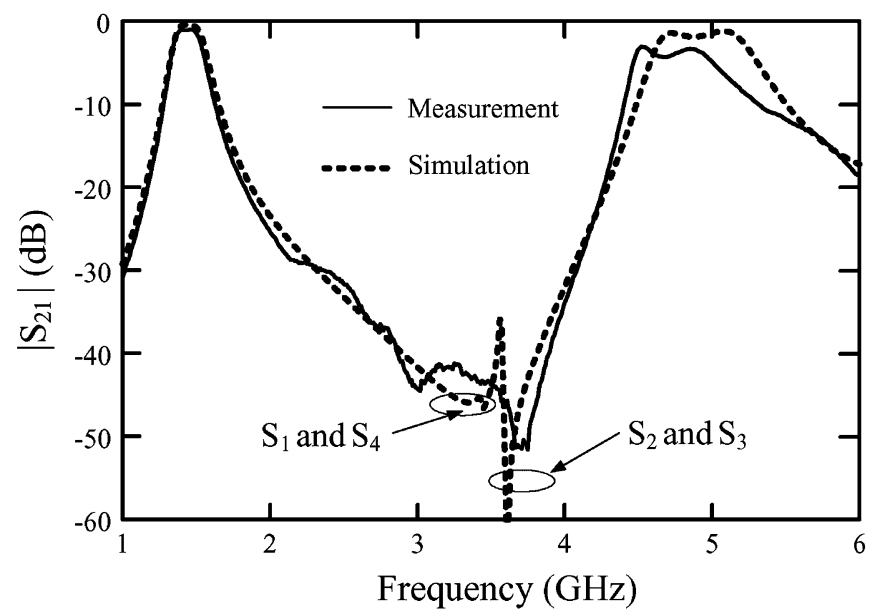

(b)

Fig. 9. Measured and simulated results of the second-order filter in Fig. 8. (a) Narrowband and (b) wideband frequency responses.

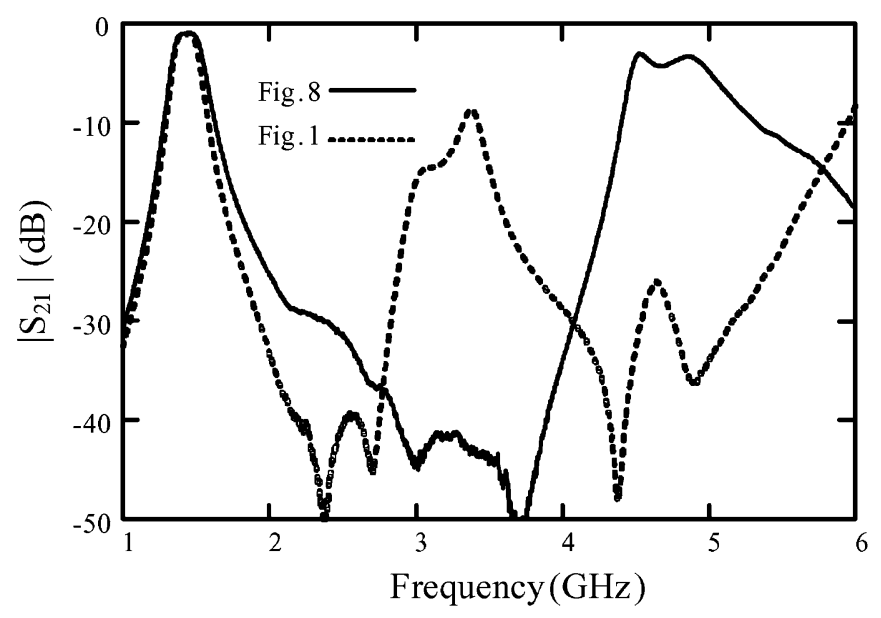

Fig. 10. Comparison of the measured responses for the filters in Figs. 1 and 8.

The implemented filter has a dimension of $0.485 \lambda \times 0.42 \lambda$ $(64.6 \mathrm{~mm} \times 56 \mathrm{~mm})$.

The measured and simulated results for the implemented fifth-order filter (Fig. 11) are shown in Fig. 12. The measured center frequency is at $1.33 \mathrm{GHz}$ and the minimum insertion loss is $2.92 \mathrm{~dB}$. Good agreement between measured and simulated
- Microstrip (top layer)

CPW (bottom layer)

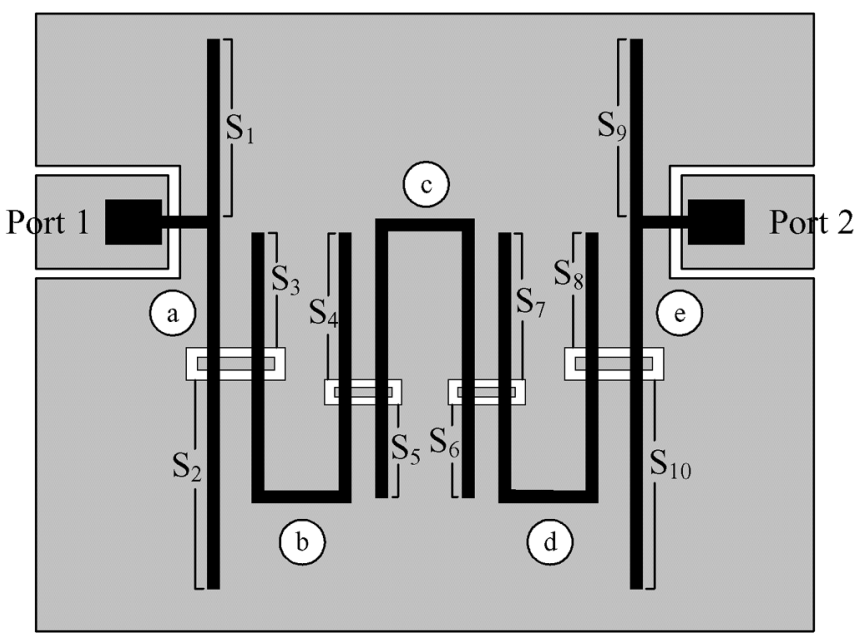

Fig. 11. Top-/bottom-layer layouts of the proposed fifth-order filter for multiple spurious suppression.

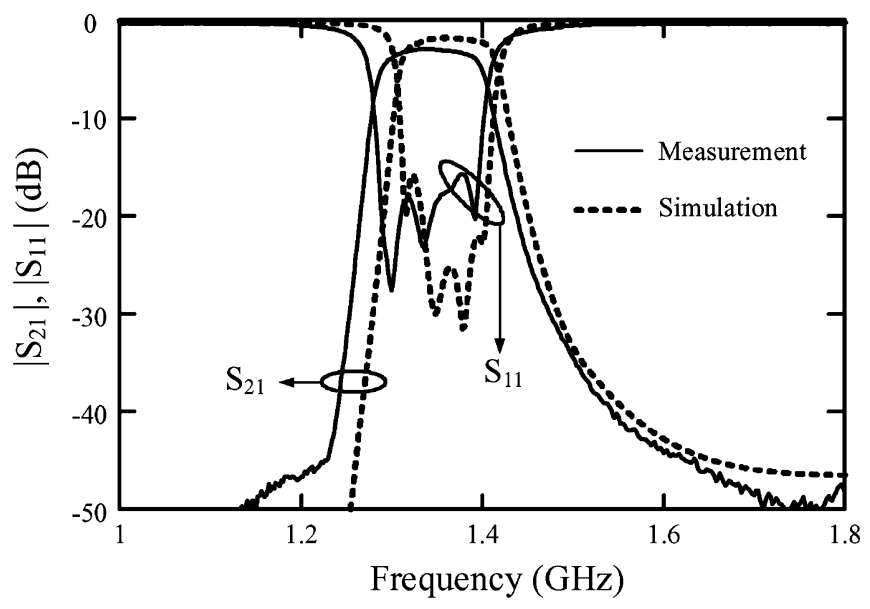

(a)

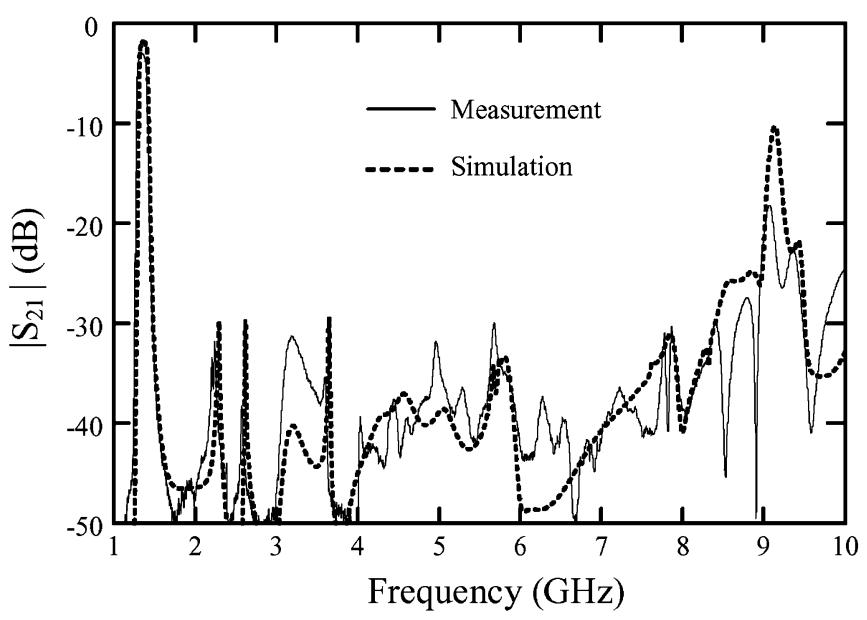

(b)

Fig. 12. Measured and simulated results of the proposed fifth-order filter in Fig. 11. (a) Narrowband and (b) wideband frequency responses.

results is observed, except for a slight frequency shift of less than $2 \%$ around passband. As shown in Fig. 12(b), the stopband 


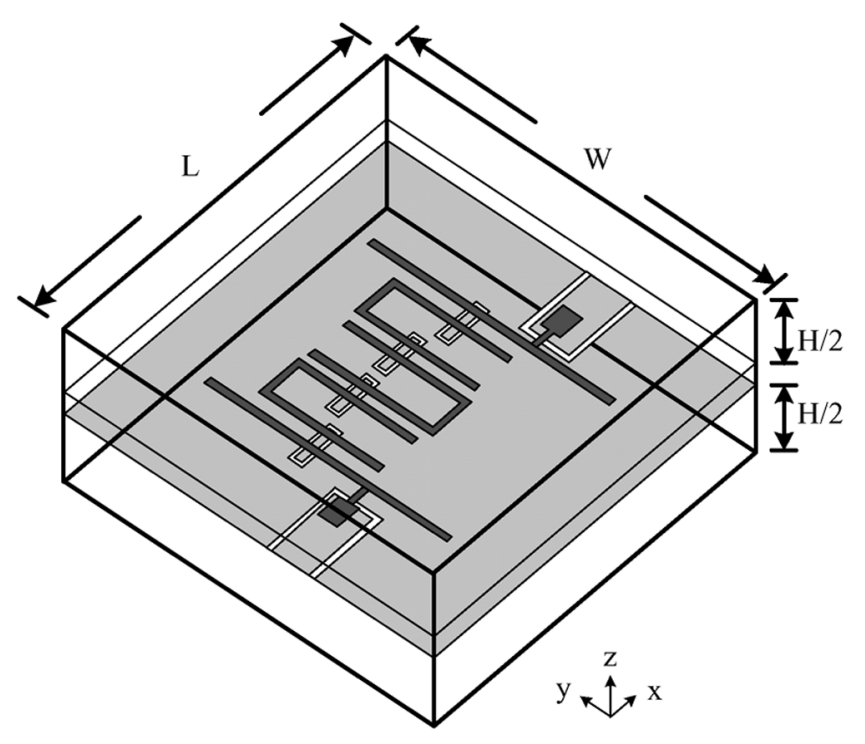

Fig. 13. Rectangular housing structure for the proposed fifth-order filter in Fig. 11. ( $W=86 \mathrm{~mm}, L=84.8 \mathrm{~mm}, H=30 \mathrm{~mm}$.)

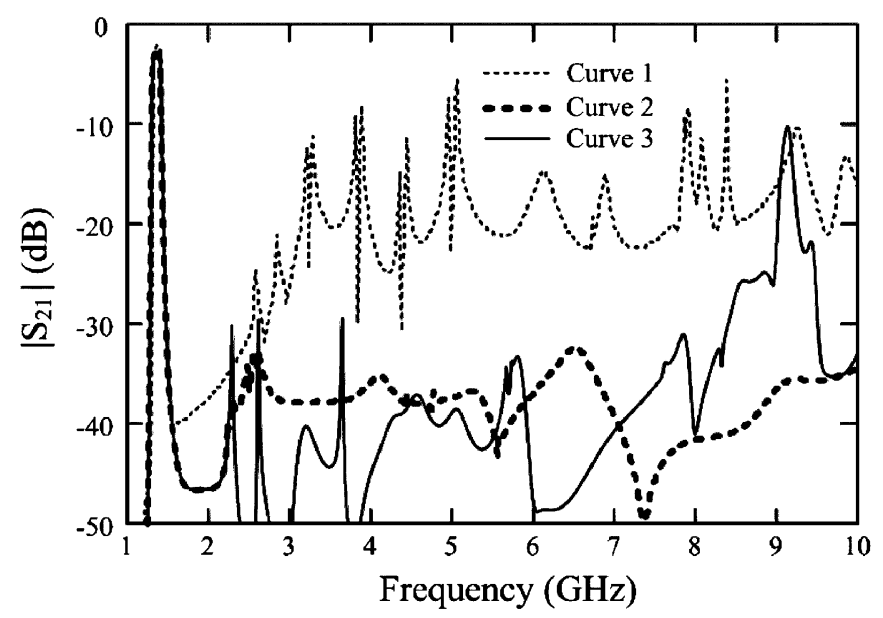

Fig. 14. Simulated results of the fifth-order filter in Fig. 11 with and without housing. (Curve 1: with housing, but without absorber, Curve 2: with housing and absorber, Curve 3: without housing.)

is extended up to $8.67 \mathrm{GHz}\left(6.52 f_{0}\right)$ with a rejection level better than $30 \mathrm{~dB}$. Note that the proposed fifth-order filter has suppressed the five spurious passbands.

To provide necessary support and to prevent unnecessary interferences, the filter is usually surrounded by a metallic housing in the system application. Physically, the housing may cause unwanted cavity resonances to degrade the spurious-suppression characteristic. Thus, the housing effect should be dealt with in the filter design phase. Note that the cavity resonances would eventually appear no matter how the housing is arranged and reshaped. Shown in Fig. 13 is a typical rectangular housing structure for the proposed fifth-order filter in Fig. 11. An approach to suppress the unwanted cavity resonances is to attach the absorbing material inside the housing walls [16]. Curve 1 of Fig. 14 exhibits the simulated result of the filter with the metallic housing (but without the absorbing material). Obviously, the spurious-suppression characteristic has been destroyed due to the cavity resonances, and extra spurious passbands are observed around the resonant frequencies of the cavity. By attaching the absorbing material on the top and bottom walls of the housing, those unwanted resonances may be suppressed, as shown in the simulated Curve 2 in Fig. 14. Although the use of absorbing material may provide a solution for suppressing the unwanted cavity resonances, the lossy absorbing material would also increase the insertion loss of the filter. As shown in Fig. 14, the insertion loss of Curve 1 is $2.2 \mathrm{~dB}$, but that of Curve 2 is $2.93 \mathrm{~dB}$ at the passband center frequency.

\section{CONCLUSION}

In this paper, the CPW-fed microstrip bandpass filters have been implemented and carefully examined with capacitively broadside-coupled structures established at I/O ports and between adjacent resonators so as to provide extra open stubs for spurious suppression. By adjusting the lengths of open stubs, multiple transmission zeros may be created and suitably distributed without increasing the circuit area so that multiple spurious passbands may effectively be suppressed and the rejection bandwidth may be extended. Specifically, a fifth-order bandpass filter, centered at $f_{0}=1.33 \mathrm{GHz}$, has been implemented and its stopband has been extended up to $8.67 \mathrm{GHz}$ $\left(6.52 f_{0}\right)$ with a rejection level better than $30 \mathrm{~dB}$.

\section{REFERENCES}

[1] M. Makimoto and S. Yamashita, "Bandpass filters using parallel coupled stripline stepped impedance resonators," IEEE Trans. Microw. Theory Tech., vol. MTT-28, no. 12, pp. 1413-1417, Dec. 1980.

[2] S. Y. Lee and C. M. Tsai, "New cross-coupled filter design using improved hairpin resonators," IEEE Trans. Microw. Theory Tech., vol. 48, no. MTT-12, pp. 2482-2490, Dec. 2000.

[3] C. M. Tsai, S. Y. Lee, and C. C. Tsai, "Performance of a planar filter using a $0^{\circ}$ feed structure," IEEE Trans. Microw. Theory Tech., vol. 50, no. 10 , pp. 2362-2367, Oct. 2002.

[4] T. Lopetegi, M. A. G. Laso, J. Hernandez, M. Bacaicoa, D. Benito, M. J. Grade, M. Sorolla, and M. Guglielmi, "New microstrip 'wigglyline' filters with spurious passband suppression," IEEE Trans. Microw. Theory Tech., vol. 49, no. 9, pp. 1593-1598, Sep. 2001.

[5] J. T. Kuo, W. H. Hsu, and W. H. Hsu, "Parallel coupled microstrip filters with suppression of harmonic response," IEEE Microw. Wireless Compon. Lett., vol. 12, no. 10, pp. 383-385, Oct. 2002.

[6] B. S. Kim, J. W. Lee, and M. S. Song, "An implementation of harmonic-suppression microstrip filters with periodic grooves," IEEE Microw. Wireless Compon. Lett., vol. 14, no. 9, pp. 413-415, Sep. 2004.

[7] S. Sun and L. Zhu, "Periodically nonuniform coupled microstrip-line filters with harmonic suppression using transmission zero reallocation," IEEE Trans. Microw. Theory Tech., vol. 53, no. 5, pp. 1817-1822, May 2005.

[8] J. T. Kuo, S. P. Chen, and M. Jiang, "Parallel-coupled microstrip filters with over-coupled end stages for suppression of spurious responses," IEEE Microw. Wireless Compon. Lett., vol. 13, no. 10, pp. 440-442, Oct. 2003.

[9] J. T. Kuo, M. Jiang, and H. J. Chang, "Design of parallel-coupled microstrip filters with suppression of spurious resonances using substrate suspension," IEEE Trans. Microw. Theory Tech., vol. 52, no. 1, pp. 83-89, Jan. 2004.

[10] M. d. C. Velazquez-Ahumada, J. Martel, and F. Medina, "Parallel coupled microstrip filters with ground-plane aperture for spurious band suppression and enhanced coupling," IEEE Trans. Microw. Theory Tech., vol. 52, no. 3, pp. 1082-1086, Mar. 2004.

[11] J. Garacia-Garacia, F. Martin, F. Falcone, J. Bonache, I. Gil, T. Lopetegi, M. A. G. Laso, M. Sorolla, and R. Marques, "Spurious passband suppression in microstrip coupled line bandpass filters by means of split ring resonators," IEEE Microw. Wireless Compon. Lett., vol. 14 , no. 9 , pp. 416-418, Sep. 2004. 
[12] S. M. Wang, C. H. Chi, M. Y. Hsieh, and C. Y. Chang, "Miniaturized spurious passband suppression microstrip filter using meandered parallel coupled lines," IEEE Trans. Microw. Theory Tech., vol. 53, no. 2, pp. 747-753, Feb. 2005.

[13] P. H. Deng, S. C. Lin, Y. S. Lin, C. H. Wang, and C. H. Chen, "Microstrip bandpass filters with dissimilar resonators for suppression of spurious responses," in Proc. 35th Microw. Eur. Conf., Paris, France, Oct. 2005, pp. 1263-1266.

[14] C. F. Chen, T. Y. Huang, and R. B. Wu, "Design of microstrip bandpass filters with multiorder spurious-mode suppression," IEEE Trans. Microw. Theory Tech., vol. 53, no. 12, pp. 3788-3793, Dec. 2005.

[15] S. C. Lin, P. H. Deng, Y. S. Lin, C. H. Wang, and C. H. Chen, "Widestopband microstrip bandpass filters using dissimilar quarter-wavelength stepped-impedance resonators," IEEE Trans. Microw. Theory Tech., vol. 54, no. 3, pp. 1011-1018, Mar. 2006.

[16] S. C. Lin, T. N. Kuo, Y. S. Lin, and C. H. Chen, "Novel coplanarwaveguide bandpass filters using loaded air-bridge enhanced capacitors and broadside-coupled transition structures for wideband spurious suppression," IEEE Trans. Microw. Theory Tech., vol. 54, no. 8, pp. 3359-3369, Aug. 2006.

[17] K. Wada and I. Awai, "Heuristic models of half-wavelength resonator bandpass filter with attenuation poles," Electron. Lett., vol. 35, no. 3, pp. 401-402, Mar. 1999.

[18] T. Ohno, K. Wada, and O. Hashimoto, "A class of a planar triplexer by manipulating multiple attenuation poles," in Proc. 34th Microw. Eur. Conf., Amsterdam, The Netherlands, Oct. 2004, pp. 625-628.

[19] J. T. Kuo and E. Shih, "Microstrip stepped impedance resonator bandpass filter with an extended optimal rejection bandwidth," IEEE Trans. Microw. Theory Tech., vol. 51, no. 5, pp. 1554-1559, May 2003.

[20] K. M. Shum, T. T. Mo, Q. Xue, and C. H. Chan, "A compact bandpass filter with two tuning transmission zeros using a CMRC resonator," IEEE Trans. Microw. Theory Tech., vol. 53, no. 3, pp. 895-900, Mar. 2005.

[21] A. Manchec, C. Quendo, I.-F. Favennec, E. Rius, and C. Person, "Synthesis of capacitive-coupled dual-behavior resonator (CCDBR) filters," IEEE Trans. Microw. Theory Tech., vol. 54, no. 6, pp. 2346-2355, Jun. 2006.

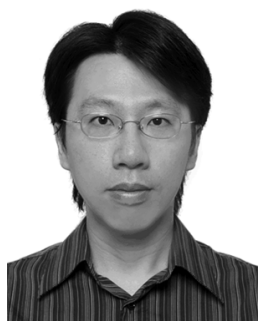

tromagnetics.

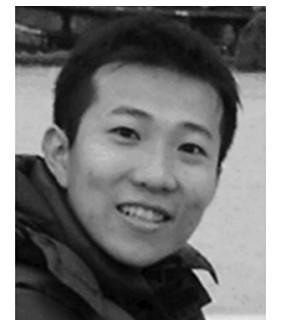

Pu-Hua Deng was born in Kaohsiung, Taiwan, R.O.C., in 1978. He received the B.S. degree in electrical engineering from National Sun Yet-Sen University, Kaohsiung, Taiwan, R.O.C., in 2002, and the M.S.E.E. and Ph.D. degrees from National Taiwan University, Taipei, Taiwan, R.O.C., in 2004 and 2006, respectively.

His research interests include the design and analysis of microwave filter circuits.

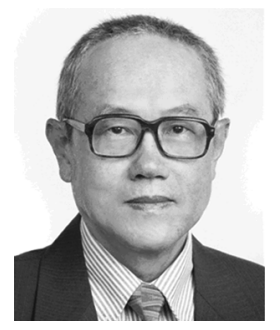

Chun Hsiung Chen (SM'88-F'96) was born in Taipei, Taiwan, R.O.C., on March 7, 1937. He received the B.S.E.E. and Ph.D. degrees in electrical engineering from National Taiwan University, Taipei, Taiwan, R.O.C., in 1960 and 1972, respectively, and the M.S.E.E. degree from National Chiao Tung University, Hsinchu, Taiwan, R.O.C., in 1962.

In 1963, he joined the Faculty of the Department of Electrical Engineering, National Taiwan University, where he is currently a Professor. From August 1982 to July 1985, he was Chairman of the Department of Electrical Engineering, National Taiwan University. From August 1992 to July 1996, he was the Director of the University Computer Center, National Taiwan University. In 1974, he was a Visiting Scholar with the Department of Electrical Engineering and Computer Sciences, University of California at Berkeley. From August 1986 to July 1987, he was a Visiting Professor with the Department of Electrical Engineering, University of Houston, Houston, TX. In 1989, 1990, and 1994, he visited the Microwave Department, Technical University of Munich, Munich, Germany, the Laboratoire d'Optique Electromagnetique, Faculte des Sciences et Techniques de Saint-Jerome, Universite d'Aix-Marseille III, Marseille, France, and the Department of Electrical Engineering, Michigan State University, East Lansing, respectively. His areas of interest include microwave circuits and computational electromagnetics. 